\title{
Widespread Multi-Organ Involvement by Lymphoplasmacytic B-Cell Lymphoma in a Patient with Multiple Comorbidities: A Case Report
}

\author{
Oluwaseyi Olayinka*, Gagandeep Kaur, Nusrat Pathan and Paul Fiedler \\ Department of Pathology and Laboratory Medicine, Danbury Hospital, Danbury, Connecticut, USA
}

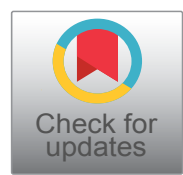

*Corresponding author: Oluwaseyi Olayinka, MD, MSc, Department of Pathology and Laboratory Medicine, Danbury Hospital, Danbury, CT-06810, USA

\begin{abstract}
Lymphoplasmacytic lymphoma (LPL) is an uncommon B-cell non-Hodgkin lymphoma in which the affected tissue, usually bone marrow, is infiltrated by small lymphocytes, plasmacytoid lymphocytes, and plasma cells. The lymph nodes, spleen and other tissues/organs in the body are less commonly infiltrated by the neoplastic cells. The clinical course of LPL is indolent although some studies report a more aggressive course and adverse outcomes especially in patients with other primary malignancies including skin cancers. We report an unusual finding of widespread multi-organ involvement by LPL in a 69-year-old male with multiple comorbid conditions and malignant melanoma. He initially presented to the hospital three years ago with hepatosplenomegaly and lymphadenopathy. Pathologic work-up including bone marrow evaluation confirmed the diagnosis of LPL. He was also diagnosed with Waldenström macroglobulinemia. Patient received chemotherapy and was well until about eight months ago when he re-presented with abdominal discomfort from a possible spontaneous bacterial peritonitis. However, during his hospital admission, he developed multiple complications including atrial fibrillation with rapid ventricular rate, persistent hypotension and decreased urinary output. He deteriorated despite active management and was transitioned to comfort care. He was later pronounced dead. Autopsy showed involvement of multiple tissues and organs including the peritoneum, parathyroid glands, heart, and brain by the patient's known B- cell lymphoma. There was extensive involvement of the bone marrow, spleen, liver and multiple lymph nodes. Histologic sections taken from the involved tissues and organs revealed infiltration by small B-cells with plasmacytoid lymphocytes and plasma cells. Sections from the scalp lesions showed in-situ and invasive malignant melanoma with involvement of the left lung.
\end{abstract}

\section{Keywords}

Lymphoplasmacytic B-cell lymphoma, Waldenström macroglobulinemia, Parathyroid gland lymphoma

\section{Introduction}

Lymphoplasmacytic lymphoma (LPL) is a neoplasm of small B lymphocytes, plasmacytoid lymphocytes, and plasma cells, commonly involving the bone marrow and sometimes the lymph nodes and spleen, which do not meet the criteria for other small B-cell lymphoid neoplasms with plasmacytic differentiation [1]. The disease is uncommon with an annual incidence of 3 to 4 cases per million people [2]. Older adults are often affected, 50 to 60 percent of whom are males [3]. Waldenström macroglobulinemia (WM), defined as LPL with bone marrow involvement and production of IgM monoclonal protein, is seen in a subset of patients with LPL. The clinical features of LPL, which includes anemia, lymphadenopathy, organomegaly, and hyperviscosity are a result of tumor infiltration and monoclonal protein production [2]. About a third of patients are asymptomatic at diagnosis [4]. The bone marrow and less commonly lymph nodes and spleen are involved by LPL. Involvement of other tissues and organs such as lungs, stomach or bowel have rarely been described. Although the cause of LPL is not fully understood, recent studies provide data supporting the role of immune related and genetic factors in the pathogenesis of the disease [5]. It is now known that

\footnotetext{
Citation: Olayinka O, Kaur G, Pathan N, Fiedler P (2019) Widespread Multi-Organ Involvement by Lymphoplasmacytic B-Cell Lymphoma in a Patient with Multiple Comorbidities: A Case Report. Int J Pathol Clin Res 5:103. doi.org/10.23937/2469-5807/1510103

Accepted: October 29, 2019: Published: October 31, 2019

Copyright: (c) 2019 Olayinka O, et al. This is an open-access article distributed under the terms of the Creative Commons Attribution License, which permits unrestricted use, distribution, and reproduction in any medium, provided the original author and source are credited.
} 
approximately 90\% of LPL or WM have MYD88 L265P mutations [1]. Mutations in CXCR4, and ARID1A have also been described [6-8]. The diagnosis of LPL is mainly based on histopathologic evaluation of involved tissues, immunophenotypic studies and exclusion of other small B cell lymphoid neoplasms with plasmacytic differentiation. The clinical course of LPL is indolent; however, some studies report a more aggressive course with poor clinical outcomes [9]. Factors such as advanced age, poor performance status, peripheral blood cytopenias, and high beta- 2 microglobulin levels have been associated with poor prognosis in patients with LPL while the presence of increased immunoblasts/transformed cells and deletion $6 \mathrm{q}$ are considered independent adverse factors [2]. The absence of an MYD88 L265P mutation has been associated with an adverse outcome [2]. There is also growing evidence of an association between non-Hodgkin lymphoma (NHL) and skin cancers. Patients with NHL have aggressive forms of skin cancer including melanomas and demonstrate worse outcomes including disease recurrence, metastasis and death [10]. LPL may transform to diffuse large B-cell lymphoma and is associated with poor survival.

\section{Case Description}

A 69-year-old male diagnosed with LPL and Walden- ström's macroglobulinemia about 3 years ago when he presented to the hospital with hepatosplenomegaly and lymphadenopathy. He had multiple chronic conditions including hypertension, hyperlipidemia, coronary artery disease and multiple skin cancers (basal cell carcinoma involving the left ear, melanoma of the back and scalp and left lower extremity lesion consistent with squamous cell carcinoma). His peripheral blood at the time showed hypochromic normocytic anemia with adequate leukocytes and platelets. Bone marrow aspirate and biopsy showed extensive involvement of the bone marrow by B-cell lymphoma with plasmacytic differentiation. The bone marrow biopsy demonstrated a markedly hypercellular marrow for age with only foci of residual trilineage hematopoiesis due to a slightly nodular and diffuse proliferation of small lymphocytes with slightly irregular nuclear membranes, clumped chromatin, inconspicuous nucleoli and scant cytoplasm. Many admixed plasma cells and plasmacytoid lymphocytes were present (Figure 1). The lymphocytic proliferation showed both paratrabecular and interstitial infiltration. The lymphomatous infiltrate showed positive immunohistochemical stains for CD20 and CD79a and was negative for CD5, Bcl-6, cyclin D1 and SOX-11 (Figure 2). CD20 demonstrated that the lymphoma comprised approximately $70-80 \%$ of the marrow cellularity while CD138 demonstrated a substantial plasma cell compo-
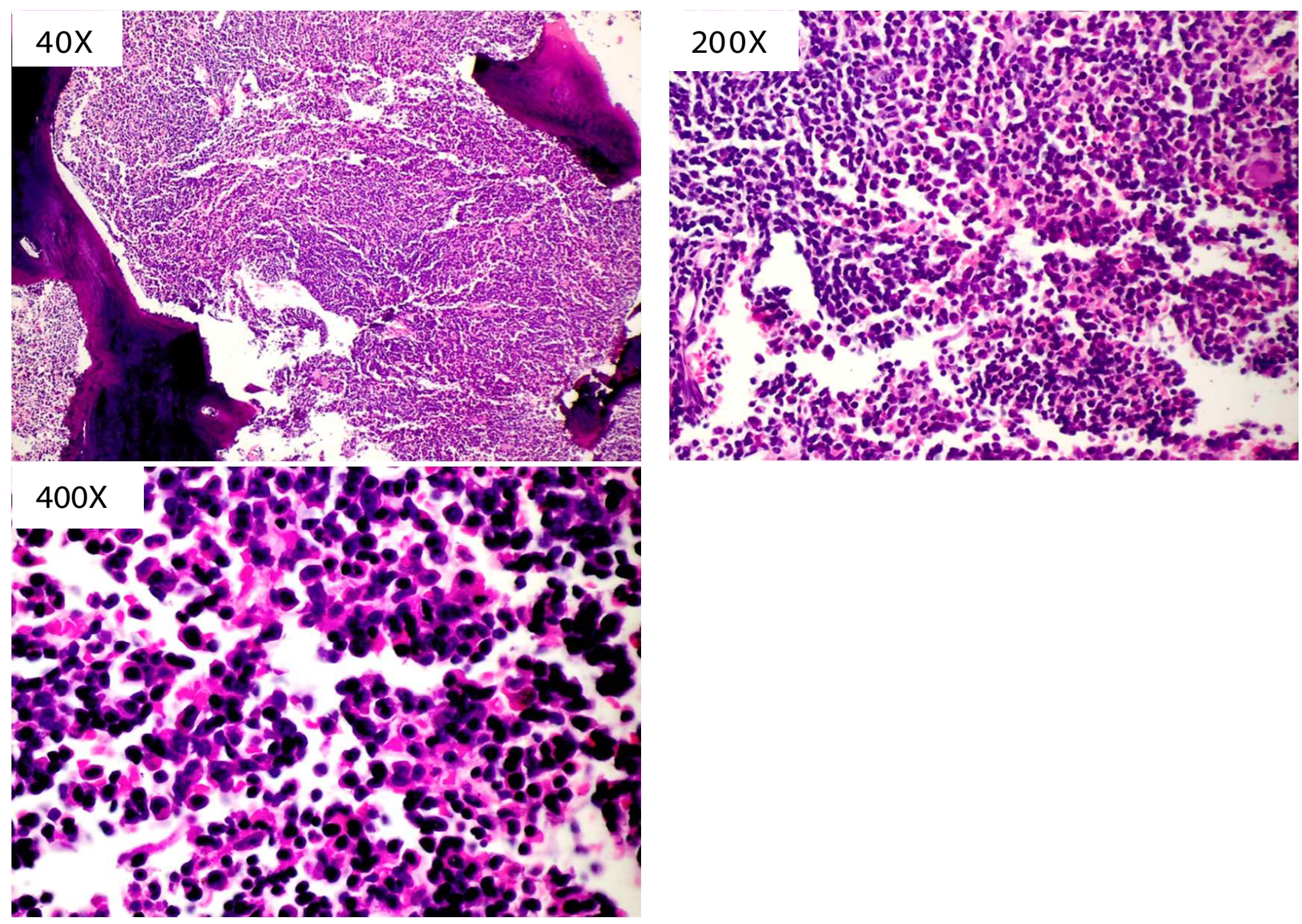

Figure 1: Hematoxylin and eosin staining demonstrating hypercellular marrow with diffuse proliferation of small lymphocytes admixed with plasma cells and plasmacytoid lymphocytes. 

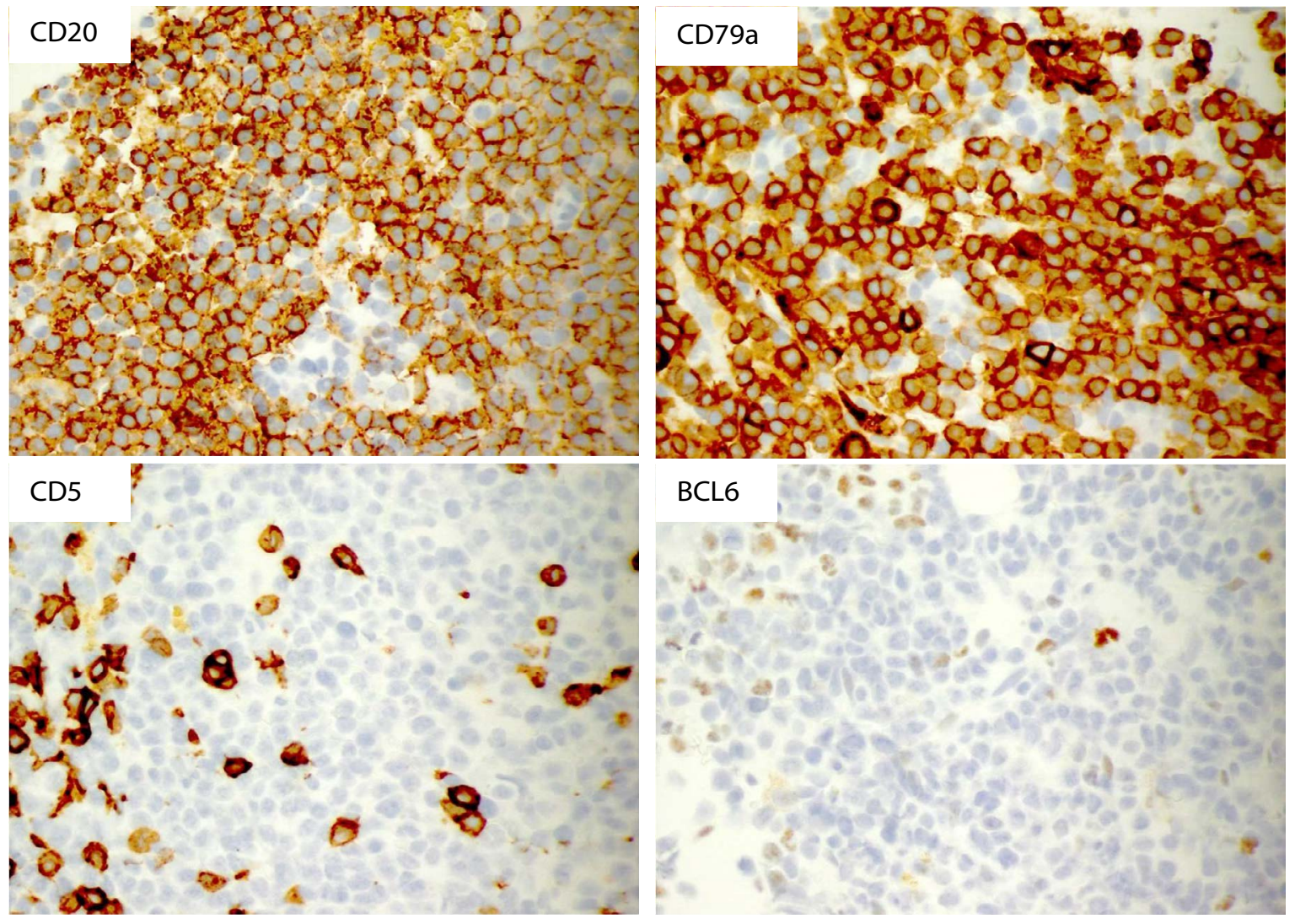

Figure 2: Immunohistochemistry showing positive staining with CD20 and CD79a and negative staining with CD5 and BCL6.
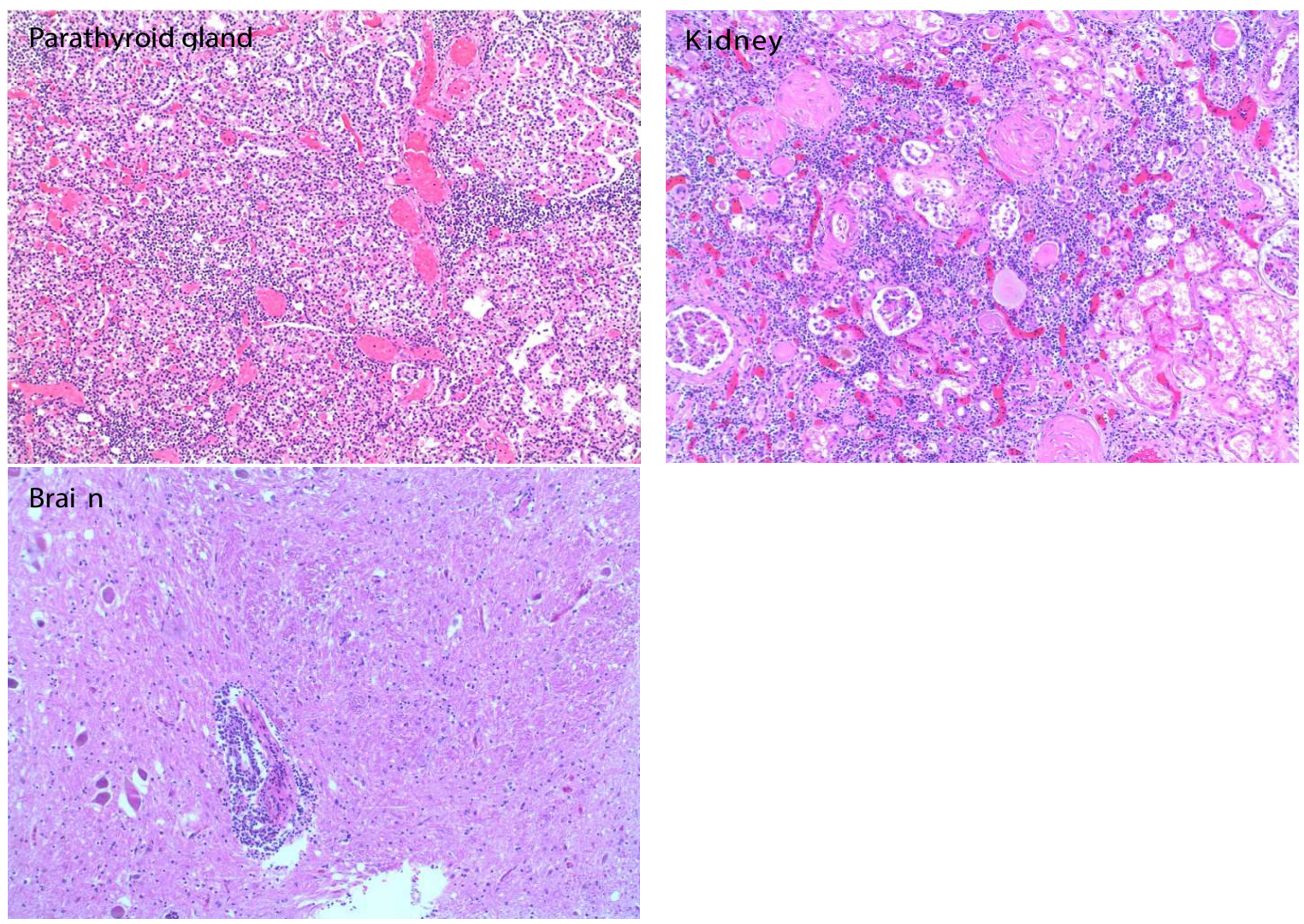

Figure 3: Hematoxylin and eosin staining showing lymphoplasmacytic infiltration of the parathyroid gland, kidneys and brain.

nent, $10-15 \%$ of the cellularity. Immunophenotyping by flow cytometry analysis showed a CD5-/CD10- monoclonal lambda restricted mature B-cell neoplasm with a small component of lambda restricted plasma cells. Serum immunofixation showed an IgM type lambda gammopathy with possible free lambda light chain. Pa- 
tient had genetic testing which revealed normal chromosomes with positivity for MYD88 mutation and negativity for MALT, BCL 6, IGH/BCL-2 and CYCLIND1/IGH by FISH.

He received systemic chemotherapy with Bendamustine and Rituximab and was doing well until about a year ago when he re-presented to the hospital with worsening weight loss and fatigue. Repeat bone marrow biopsy was similar to the initial biopsy with many of the plasma cells showing atypical features including binucleation, high NC ratio and prominent nucleoli. He was subsequently scheduled for Ibrutinib therapy.
While awaiting therapy, patient developed a right hip fracture secondary to a mechanical fall. He was treated with open reduction and internal fixation with rehabilitation. During this period, he was noted to have cholecystitis requiring a percutaneous drain, pneumonia and Clostridium difficile infection for which he was treated and got better. Patient later commenced Ibrutinib therapy following which he felt less fatigued with a stable weight until about eight months ago when he developed abdominal pain of sudden onset. He reported back to the hospital where he was noted to have ascites. He was admitted and worked
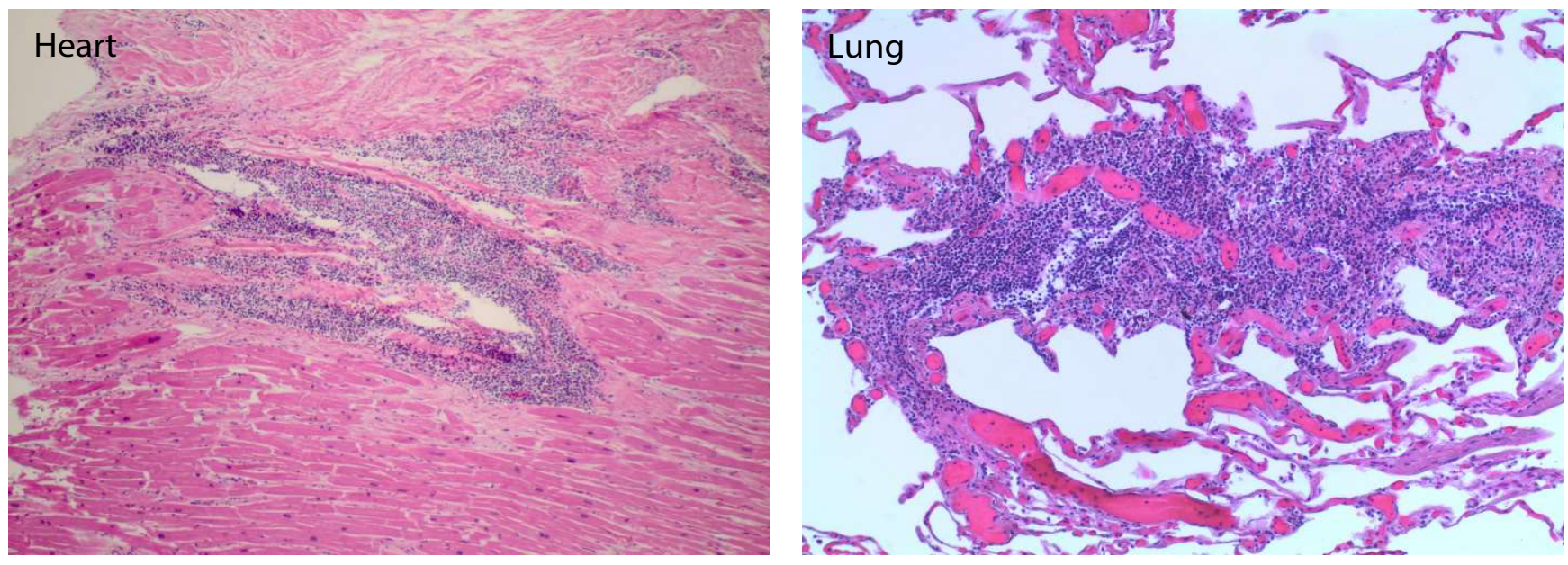

Figure 4: Hematoxylin and eosin staining showing patchy involvement of the heart and lungs by lymphoma.

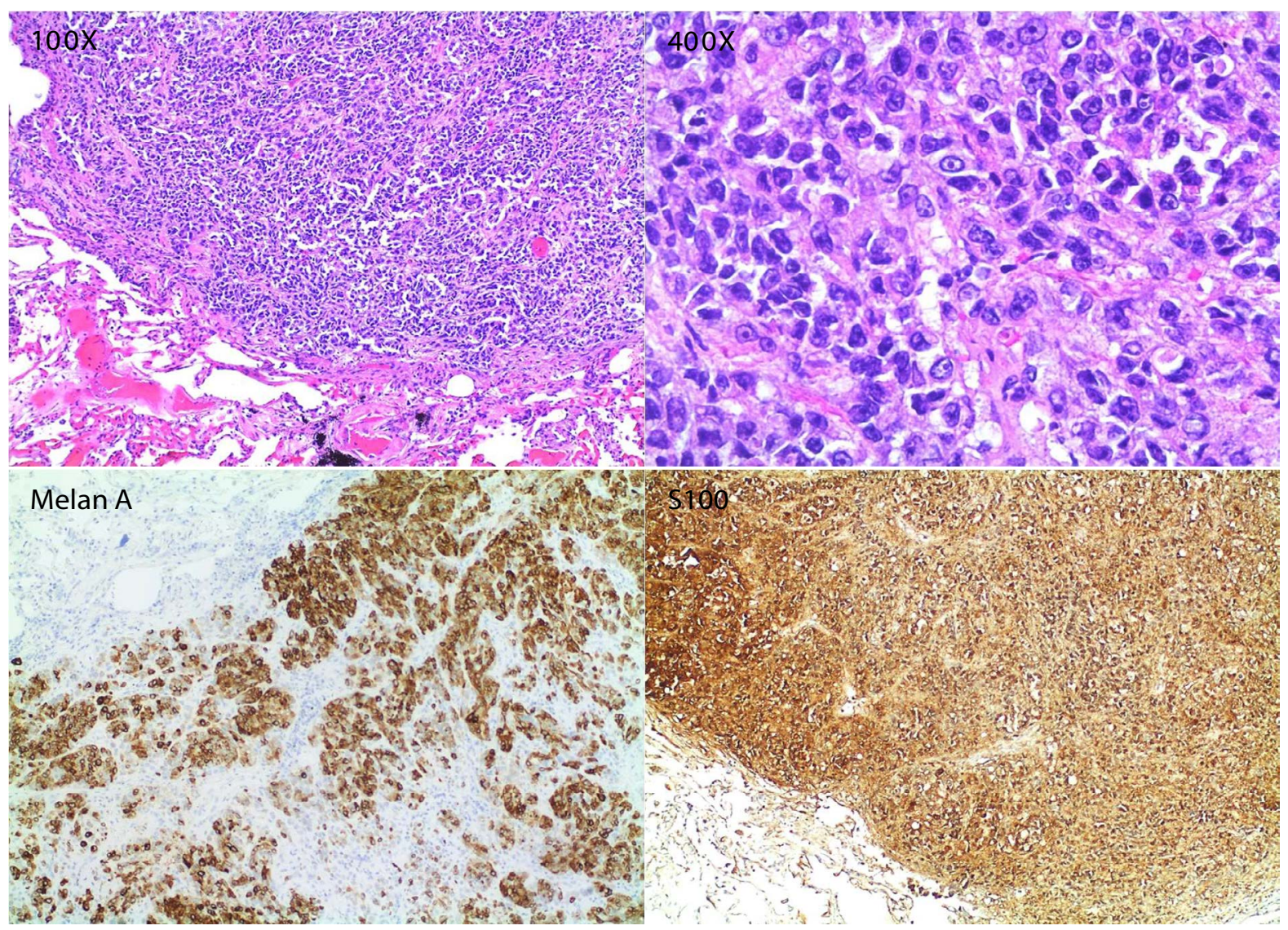

Figure 5: Hematoxylin and eosin staining and Immunohistochemistry of the left lung showing malignant cells with features consistent with metastatic melanoma. Tumor cells show positive Melan A and S-100 staining. 
up for possible spontaneous bacterial peritonitis. His ascitic fluid was positive for methicillin-resistant staphylococcus aureus. Unfortunately, during this admission patient developed multiple complications including atrial fibrillation with rapid ventricular rate, persistent asymptomatic hypotension and decreased urinary output. He continued to deteriorate despite extensive resuscitative measures. Goals of care were discussed with the patient and his family. Given the patient's poor clinical outcome, he was transitioned to comfort care. He was later pronounced dead.

Autopsy showed widespread involvement of multiple tissues and organs by lymphoma with metastatic malignant melanoma. There was extensive involvement of the bone marrow, spleen, liver and multiple lymph nodes by neoplastic lymphoplasmacytic infiltrate consisting of small mature lymphocytes, plasmacytoid lymphocytes and plasma cells. The parathyroid glands, kidneys and brain were also involved (Figure 3). There was patchy involvement of myocardium, pericardium, and both lungs by the lymphoma (Figure 4). In addition, sections of the upper and lower lobes of the left lung showed malignant cells with morphologic and immunophenotypic features consistent with metastatic melanoma (Figure 5). The tumor cells were positive for Melan A and S-100 with patchy staining for HMB-45. Cytokeratin AE1/AE3 and TTF-1 stains were negative. The abdominal cavity revealed more than a liter of ascitic fluid and extensive peritoneal adhesions with acute necrotizing peritonitis and involvement by lymphoma. Gross examination of the skin showed multiple lesions in the scalp, forehead and upper extremities at different stages of healing. Histologically, sections from the scalp lesions showed in-situ and invasive malignant melanoma.

\section{Conclusions}

Studies on multisystemic involvement by LPL have rarely been published. We report autopsy findings of widespread involvement of multiple tissues and organs by lymphoma in a 69 -year-old male who developed both melanoma and non-melanoma skin cancer in the setting of LPL/Waldenström's macroglobulinemia. Histologic sections showed infiltration of the bone marrow, spleen, liver and lymph nodes by small B-cells with plasmacytoid lymphocytes and plasma cells with rare involvement of the kidney, heart, brain and parathyroid glands. He had in-situ and invasive malignant melanoma with metastasis to the lungs which also showed involvement by lymphoma. There was no evidence of transformation to large B-cell lymphoma. Although the most likely cause of death in this patient is his widespread known B-cell lymphoma, other factors such as the presence of an already compromised cardiac function and skin cancer may have contributed to his death. Studies have shown that patients with NHL who later develop skin cancer and those with skin cancer who develop NHL have worse clinical outcomes evidenced by increased rates of local recurrence, metastasis and death [10]. Impairment of the immune system, a proposed pathogenic factor in the association between NHL and skin cancer, has been linked to the poor outcomes seen in these patients. It is possible that the presence of skin cancer further weakens an already suppressed immune system, leading to widespread disease/metastasis. However, more studies are needed to further clarify the relationship and investigate outcomes in patients with $\mathrm{NHL}$ and skin cancer.

\section{References}

1. Swerdlow SH, Campo E, Pileri SA, Harris NL, Stein H, et al. (2016) The 2016 revision of the World Health Organization classification of lymphoid neoplasms. Blood 127: 23752390.

2. Hatem Kaseb, Prerna Mewawalla (2019) Cancer, Lymphoplasmacytic Lymphoma (Waldenstrom Macroglobulinemia). StatPearls.

3. Arnold S Freedman, Jon C Aster (2018) Clinical manifestations, pathologic features, and diagnosis of lymphoplasmacytic lymphoma. Uptodate.

4. García-Sanz R, Montoto S, Torrequebrada A, De Coca AG, Petit J, et al. (2001) Waldenström macroglobulinaemia: Presenting features and outcome in a series with 217 cases. $\mathrm{Br} \mathrm{J}$ Haematol 115: 575-582.

5. Naderi N, Yang DT (2013) Lymphoplasmacytic lymphoma and Waldenström macroglobulinemia. Arch Pathol Lab Med 137: 580-585.

6. Hunter ZR, Xu L, Yang G, Zhou Y, Liu X, et al. (2014) The genomic landscape of Waldenström macroglobulinemia is characterized by highly recurring MYD88 and WHIM-like CXCR4 mutations, and small somatic deletions associated with B-cell lymphomagenesis. Blood 123: 1637-1646.

7. Treon SP, Xu L, Yang G, Zhou Y, Liu X, et al. (2012) MYD88 L265P somatic mutation in Waldenström's macroglobulinemia. N Engl J Med 367: 826-833.

8. Vinarkar S, Arora N, Chowdhury SS, Saha K, Pal B, et al. (2019) MYD88 and CXCR4 Mutation Profiling in Lymphoplasmacytic Lymphoma/Waldenstrom's Macroglobulinaemia. Indian Journal of Hematology and Blood Transfusion 35: 57-65.

9. Otley CC (2006) Non-Hodgkin lymphoma and skin cancer: A dangerous combination. Australasian Journal of Dermatology 47: 231-236.

10. Brewer JD (2010) Skin cancer in patients with non-Hodgkin's lymphoma. Expert Review of Dermatology 5: 525533.

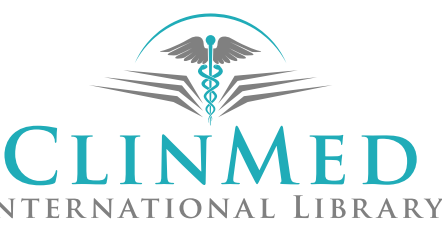

